\section{Energie der Strahlung}

Zur Bestimmung d. Energie der ausgesandten Strahlung konnte wegen der kurzen Halbwertszeit nur die Absorptionsmethode Verwendung finden. Für die Herstellung genügend materiefreier starker ${ }^{55} \mathrm{Cr}$-Präparate sind die Fällungen aus bestrahltem Mangan zu zeitraubend. Die intensivsten Präparate wurden daher mit Hilfe eines SzilardChalmers-Verfahrens erhalten.

$\mathrm{Zu}$ diesem $\mathrm{Zwecke}$ wurden $30-50 \mathrm{~g} \mathrm{~K}_{2} \mathrm{CrO}_{4}$ in Salzform bestrahlt, in heiBem Wasser gelöst, mit einigen $\mathrm{mg} \mathrm{FeCl}_{3}$ als Träger versetzt, aufgekocht, auf $60^{\circ}$ abgekühilt und nach $1-2 \mathrm{~min}$ abfiltriert. Der Niederschlag enthielt etwa $30 \%$ der gesamten Chrom-Aktivität, was zeigt, daß etwa dieser Anteil nach der Neutroneneinfangreaktion dreiwertig vorliegt und sich so abtrennen läßt ${ }^{11}$.

Die erhaltene Absorptionskurve zeigt Abb. 1, Kurve C. Durch Vergleich mit einer in derselben Geometrie aufgenommenen Absorptionskurve des ${ }^{104} \mathrm{Rh}$ (Kurve D) ergibt sich $E_{\max }=2,85 \mathrm{MeV}$ für ${ }^{55} \mathrm{Cr}$. Im Magnetfeld konnte noch festgestellt wer-

11 Über die genaueren chemischen Befunde, in welchen Wertigkeiten das aktive Chrom durch die ver-

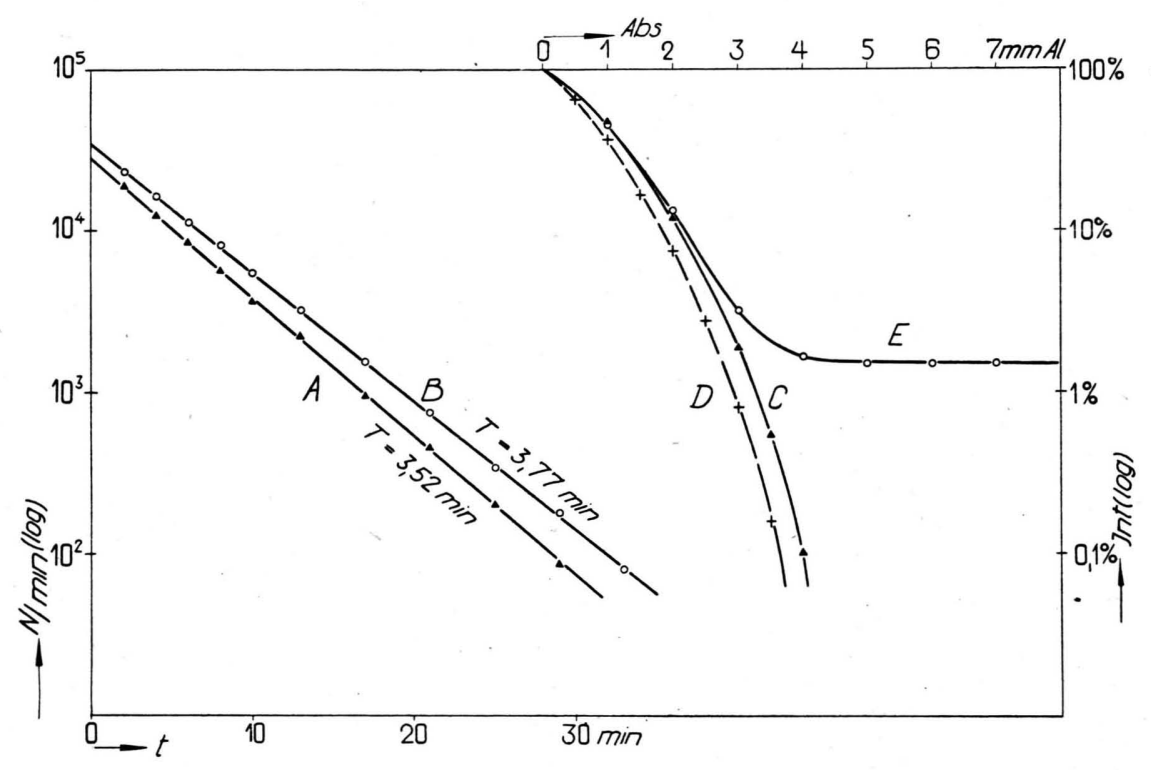

Abb. 1. A: Zeitlicher Abfall des ${ }^{55} \mathrm{Cr}$. B: Zeitlicher Abfall des ${ }^{52} \mathrm{~V}$. C: Absorptionskurve in Aluminium der $\beta$-Strahlen des ${ }^{55} \mathrm{Cr}$. D: desgl. von ${ }^{104} \mathrm{Rh}$. E: desgl. von ${ }^{52} \mathrm{~V}$.

den, daß es sich um $\beta$-Strahlen handelt. $\gamma$-Strahlen sind keine vorhanden. Zum Vergleich ist noch in Abb. 1, Kurve E, die entsprechende Absorptionskurve der Strahlung des ${ }^{52} \mathrm{~V}$ eingezeichnet, die zunächst einen sehr ähnlichen Verlauf hat, aber oberhalb von etwa $4 \mathrm{~mm}$ Aluminium einen starken $\gamma$ Untergrund aufweist. Da der $\gamma$-Untergrund des ${ }^{52} \mathrm{~V}$ mindestens die 15-fache Intensität hat wie beim ${ }^{55} \mathrm{Cr}$, bietet diese Tatsache noch eine weitere physikalische Unterscheidungsmöglichkeit der beiden Aktivitäten.

schiedenen Kernprozesse gebildet wird, soll an anderer Stelle berichtet werden.

\title{
Das chemische Isotopentrennverfahren als Rektifikationsproblem
}

\author{
Von E. W. Becker und K. Bier \\ Aus dem Physikalischen Institut der Universität Marburg a. d. Lahn \\ (Z. Naturforschg. 7 a, 651-664 [1952]; eingegangen am 4. Juni 1952)
}

\begin{abstract}
Es wird eine Methode zur Bestimmung optimaler Betriebsbedingungen für Einzelkolonnen und Kolonnen-Kaskaden am Beispiel der ${ }^{13} \mathrm{C}$-Anreicherung entwickelt. Die Abhängigkeit der Trenneigenschaften der Füllkörperkolonnen von den interessierenden Versuchsparametern wird in 60 Einzelversuchen experimentell bestimmt. Durch ein besonderes Aktivierungsverfahren kann dabei die Trennschärfe der Kolonnen erheblich verbessert werden. Mit den experimentell gewonnenen Daten wird auf Grund der abgeleiteten Formeln eine vierstufige Trennkaskade für eine Produktionsleistung von 0,18 Mol Kohlenstoff pro Tag mit $12 \%{ }^{13} \mathrm{C}$ berechnet. Die praktische Ausführung dieser Anlage wird in der folgenden Arbeit beschrieben.
\end{abstract}


$\mathrm{D}$ as von Urey und Mitarbeitern ${ }^{1}$ entwickelte chemische Austauschverfahren ist wegen seines hohen Materialdurchsatzes und geringen Energieverbrauches zur industriellen Anreicherung der biologisch wichtigen Isotope ${ }^{13} \mathrm{C},{ }^{15} \mathrm{~N}$ und ${ }^{34} \mathrm{~S}$ besonders geeignet. Bei der Planung einer größeren Austauschanlage ergibt sich die Frage, wie eine vorgegebene Trennaufgabe mit möglichst geringem Chemikalienaufwand und möglichst kurzer Anlaufzeit bewältigt werden kann. Obwohl mehrere theoretische Arbeiten über Optimalprinzipien bei Isotopentrennanlagen vorliegen, läßt sich diese Frage nur unvollständig beantworten, da sich diese Arbeiten auf Trennmethoden beziehen, die mit dem Austauschverfahren nicht unmittelbar vergleichbar sind ${ }^{2}$, oder das Problem in so allgemeiner Form behandeln, daß eine Spezialisierung Schwierigkeiten bereitet ${ }^{3}$. AuBerdem fehlt eine breitere experimentelle Untersuchung über die Abhängigkeit der Kolonneneigenschaften von den Versuohsbedingungen, ohne die eine explizite Berechnung von Austauschanlagen nicht möglich ist.

In der vorliegenden Arbeit haben wir die experimentellen und theoretischen Unterlagen so ergänzt, daß die Abmessungen und Betriebsbedingungen einer für eine vorgegebene Trennaufgabe ,optimalen" Austauschanlage vollständig festgelegt werden können. Unsere Untersuchungen beziehen sich auf die Anreicherung von ${ }^{13} \mathrm{C}$, das im natürlichen Kohlenstoff zu 1,1\% enthalten ist. Das Ergebnis läßt sich jedoch mit geringen Einschränkungen auch auf ${ }^{15} \mathrm{~N}$ und ${ }^{34} \mathrm{~S}$ übertragen.

Der erste Teil der Arbeit behandelt die Trenneigenschaften von Einzelkolonnen. Als Maß für die Leistungsfähigkeit wird ein Trennvermögen definiert, dessen Abhängigkeit von den Betriebsbedingungen wie Gasdruck, Flüssigkeitsbelastung, Konzentration der Lösung, Form und Oberflächenzustand der Füllkörper experimentell untersucht wird. Auf Grund dieser Versuche und der theoretischen Ermittlung eines günstigsten Entnahmeverhältnisses können die Bedingungen angegeben werden,

${ }^{1}$ H. G. Thode u. H. C. Urey, J. chem. Physics 7, 34 [1939]. C. A. Hutchinson, D. W. Stewart u. H. C. Urey, J. chem. Physics 8, 532 [1940].

${ }^{2}$ Vgl. z. B. die Zusammenfassung von R. C. J ones u. W. H. Furry über das Trennrohr (Rev. mod. Physics 18, 151 [1946]) oder die von M. Benedict über Gasdiffusion (Kirk-Othmer, Bd. 5, S. 76).

${ }^{3}$ In einer Arbeit von K. Cohen (The Science and Engineering of Nuclear Power, Bd. 2, Addison-Wesley Press, 1949, S. 19) wird z. B. die ideale Durchsatzfunktion für eine allgemeine, aus Elementen mit sehr unter denen eine Einzelkolonne für die Isotopentrennung optimal arbeitet.

Der zweite Teil behandelt die Kolonnenstaffelung, die beim Austauschverfahren vor allem zur Verminderung der Einstellzeit von Bedeutung ist. Es werden Formeln abgeleitet, die bei vorgegebener Trennleistung die Dimensionen einer aus ,,optimalen“ Einzelkolonnen zusammengesetzten günstigsten Trennkaskade festlegen. Als Zahlenbeispiel wird mit den im ersten Teil gewonnenen experimentellen Unterlagen eine ,optimale“ Trennkaskade berechnet, die im Dauerbetrieb pro Tag 0,18 Mol Kohlenstoff mit $12 \%{ }^{13} \mathrm{C}$ liefern soll.

Die technische Ausführung dieser Anlage und die mit ihr gewonnenen Erfahrungen werden in der folgenden Arbeit beschrieben.

\section{A. Die Einzelkolonne}

\section{Das Anreicherungsprinzip}

Die hier behandelte chemische Anreicherung des schweren Kohlenstoffisotops basiert auf der geringfügigen Isotopenverschiebung im Gleichgewicht

$$
\mathrm{H}{ }^{13} \mathrm{CN}+{ }^{12} \mathrm{CN}^{\prime} \rightleftharpoons \mathrm{H}^{12} \mathrm{CN}+{ }^{13} \mathrm{CN}^{\prime},
$$

das durch ein Zweiphasen-System aus wäßriger NaCNLösung und gasförmiger Blausäure verwirklicht wird. Bei der auf Urey und Mitarbeiter ${ }^{1}$ zurückgehenden Anreicherungsmethode wird der chemische Unterschied der Kohlenstoffisotope, der sich für die Reaktion (1) durch das Massenwirkungsgesetz

$$
\frac{\left[\mathrm{H}^{12} \mathrm{CN}\right]_{\mathrm{gas}} \cdot\left[{ }^{13} \mathrm{CN}^{\prime}\right]_{\mathrm{aq}}}{\left[\mathrm{H}^{13} \mathrm{CN}\right]_{\mathrm{gas}} \cdot\left[{ }^{12} \mathrm{CN}^{\prime}\right]_{\mathrm{aq}}}=\alpha \approx 0,97
$$

beschreiben läßt, in einem Gegenstromverfahren vervielfacht. Dazu wird Blausäure mit natürlicher Isotopenzusammensetzung von unten in eine Füllkörpersäule eingeblasen und am oberen Ende der Kolonne durch Natronlauge quantitativ in $\mathrm{NaCN}$ übergeführt. Die NaCN-Lösung läuft im Gegenstrom zur gasförmigen Blausäure über die Füllkörper nach unten, wobei sich der durch (2) charakterisierte Elementareffekt rektifikationsartig vervielfacht. Da das schwere Kohlenstoffisotop nach (2) etwas bevorzugt in der Gasphase vorhanden ist, reichert es sich am oberen Ende der Kolonne an. Im stationären Zustand liegt eine mit steigender Rohrlänge zunehmende Isotopenverschiebung vor, die zweckmäßig durch den ,, Anreicherungsfaktor“"

kleinem Anreicherungsfaktor bestehende Trennkaskade berechnet. Die in dieser Arbeit vorkommenden Begriffe ,,value function" und ,,separating power" haben eine etwas andere Bedeutung als unsere Bezeichnungen ,Wertfunktion" und ,Trennvermögen". Vgl. auch: K. Cohen, The Theory of Isotope Separation as applied to the large Scale Production of $U^{235}$. New York 1951, Mc. Graw Hill Book Comp. Wegen der Verhältnisse bei Einzelkolonnen vgl. ${ }^{3 \mathrm{a}}$.

${ }^{3 a}$ K. Cohen, J. chem. Physics 8, 588 [1940]. 


$$
A=\frac{n_{\mathrm{o}}\left(1-n_{\mathrm{u}}\right)}{n_{\mathrm{u}}\left(1-n_{\mathrm{o}}\right)} .
$$

charakterisiert wird. $\left(n_{0}, n_{\mathrm{u}}=\right.$ Molenbruch des schweren Isotops am oberen bzw. unteren Rohrende.)

Zur Isotopengewinnung entnimmt man am oberen Ende des Rohres aus dem Blausäurestrom der Stärke $l$ (Mol/Zeiteinheit) einen kleinen Stromanteil der Stärke $p$. Bedeutet $L$ die Stromstärke des austauschfähigen Materials in der Flüssigkeit, so gilt stets

$$
L=l-p \text {. }
$$

$L$ und $l$ werden im folgenden auch als Flüssigkeitsbzw. Gas-,,Durchsatz" bezeichnet".

\section{Die Wertfunktion und das optimale Entnahmeverhältnis}

Bei der Entnahme sinkt die Anreicherung der Kolonne ab, und zwar um so mehr, je größer das Entnahmeverhältnis $\pi=p / l$ gewählt wird. Für eine Kolonne mit vorgegebenem $A$ und $L$ ( $A=$ Anreicherungsfaktor im stationären Zustand ohne Entnahme) wird der Zusammenhang zwischen dem Anreicherungsfaktor im stationären Entnahmezustand

$$
a=\frac{n_{\mathrm{p}}\left(1-n_{\mathrm{u}}\right)}{n_{\mathrm{u}}\left(1-n_{\mathrm{p}}\right)}
$$

$\left(n_{\mathrm{p}}=\right.$ Molenbruch des schweren Isotops im Produktionsgas) und dem Entnahmeverhältnis $\pi$ durch Gl. (6) geliefert, die wir einer Arbeit von Cohen ${ }^{3 a}$ entnehmen

$$
a=\frac{(1-\pi) \varepsilon+\pi}{(1-\pi) \varepsilon \cdot A^{-\left(1+\frac{\alpha \pi}{\varepsilon}\right)}+\pi} .
$$

$(\varepsilon=1-\alpha=$ Elementareffekt der Austauschreaktion $^{5}$.)

Unter Berücksichtigung dieses Sachverhaltes läßt sich eine gegebene Trennaufgabe, die durch eine bestimmte Entnahmestromstärke $p$ und einen bestimmten Anreicherungsfaktor $a$ charakterisiert ist, auf verschiedene Weise lösen: Unter Konstanthal-

4 Das hier angegebene Schema einer Trennkolonne für ${ }^{13} \mathrm{C}$ ist ohne weiteres auf die Stickstofftrennung zu übertragen, die mit dem Gleichgewicht zwischen gasförmigem Ammoniak und Ammoniumnitratlösung arbeitet, bei dem sich das ${ }^{15} \mathrm{~N}$ bekanntlich in der flüssigen Phase anreichert. Man hat dazu lediglich die Bedeutung von ,Gas“" und ,Flüssigkeit" sowie von ,,oben“" und ,,unten" zu vertauschen. Es bedeutet dann $L$ die Ammoniak- und $l$ die Ammoniumnitrat-Stromstärke, $n_{\mathrm{u}}$ ist der Molenbruch des ${ }^{15} \mathrm{~N}$ in der Ammoniumnitratlösung (von natürlicher Isotopenzusammensetzung), die am oberen Ende in die Kolonne eintritt. Entsprechende Verhältnisse liegen bei der Anreicherung des ${ }^{34} \mathrm{~S}$ vor.

5 In die Formel ist ein effektiver Elementareffekt $\varepsilon$ einzusetzen, der wegen der in der Flüssigkeit gelösten Blausäure etwas kleiner ist als der in Gl. (2) für die tung der ,,spezifischen Kolonnenbelastung“ $\sigma=$ $L / Q(Q=$ Kolonnenquerschnitt) und aller-übrigen Betriebsbedingungen wie Gasdruck, Konzentration der Lösung, Art und Oberflächenbeschaffenheit der Füllkörper usw. kann man einmal den Durchsatz $L$ relativ gro $\beta$ wählen, um zu einem kleinen Entnahmeverhältnis zu kommen; dann wird sich der gewünschte Anreicherungsfaktor $a$ mit verhältnismäßig kleiner Kolonnenlänge erzielen lassen. Man kann jedoch auch den Durchsatz möglichst klein halten, etwa um Chemikalien zu sparen; dann steigt die Kolonnenlänge entsprechend an. Die Frage nach dem für die gegebene Trennaufgabe ,optimalen“" Durchsatz ist gleichbedeutend mit der Frage, welches Entnahmeverhältnis für eine gegebene Kolonne mit festem $L$ und $A$ am günstigsten ist.

Beim Aufstellen einer Optimalbedingung für $\pi$ muß man berücksichtigen, daß der Wert des produzierten Materials einmal von der Isotopenmenge abhängt, die es im Überschuß über der Normalkonzentration enthält, und zum anderen von der erzielten Anreicherung ( $a-1)$. Es liegt nun nahe, beide Gesichtspunkte in gleicher Weise $\mathrm{zu}$ bewerten und demzufolge die Mengenangabe und die Anreicherung multiplikativ in einer ,Wertfunktion“ zu vereinigen, deren Maximum eine ,optimale“ Entnahmeart definiert.

Die Isotopenmenge, die in dem pro Zeiteinheit produzierten Material im Überschuß über der Normalkonzentration enthalten ist, beträgt ${ }^{6}$

$$
\tau=p\left(n_{\mathrm{p}}-n_{\mathrm{u}}\right),
$$

oder unter Verwendung von (5)

$$
\tau=p(a-1) \frac{n_{\mathrm{u}}\left(1-n_{\mathrm{u}}\right)}{1+(a-1) n_{\mathrm{u}}} .
$$

Bezieht man $\tau$ auf den eingesetzten Blausäurestrom $l$ und damit auf den Chemikalienverbrauch, so erhält man für die Wertfunktion ${ }^{7}$

reine Ionenreaktion angegebene Wert (vgl. Abschnitt A 5a). Aus (6) erkennt man, daß für $\pi \ll 1 \mathrm{der}$ Anreicherungsfaktor $a$ bei festem $A$ praktisch nur von $\pi / \varepsilon$ abhängt; diese Tatsache erleichtert die Übertragung der im folgenden gewonnenen Ergebnisse auf andere Austauschreaktionen, $d$. h. auf andere $\varepsilon$-Werte. Wegen des Gültigkeitsbereiches von (6) vgl. ${ }^{20}$.

${ }^{6}$ Gl. (7) beschreibt den auf den stationären Zustand bezogenen Spezialfall des ,Transportes“،

$$
\tau(z)=p\left(n(z)-n_{\mathrm{u}}\right)+L(n(z)-N(z))
$$

[n $(z)$ bzw. $N(z)=$ Molenbruch des ${ }^{13} \mathrm{C}$ an der Stelle $z$ im Gas bzw. in der Flüssigkeit].

${ }^{7}$ Wenn $n_{\mathrm{u}} \ll 1$ ist, wie z. B. beim ${ }^{13} \mathrm{C}$ und ${ }^{15} \mathrm{~N}$, kann man für $a \lesssim 10 \tau \approx p(a-1) n_{\mathrm{u}}$ und $W \approx \pi(a-1)^{2} n_{\mathrm{u}}$ setzen. 


$$
W=\frac{\tau}{l}(a-1)=\pi(a-1)^{2} \frac{n_{\mathrm{u}}\left(1-n_{\mathrm{u}}\right)}{1+(a-1) n_{\mathrm{u}}} .
$$

Daraus ergibt sich mit Hilfe des Zusammenhanges zwischen $a$ und $\pi$ nach (6) der in Abb. 1 dargestellte Verlauf von $W(\pi)$. Die Wertfunktion besitzt demnach für jeden Wert des Parameters $A$ ein Maximum bei einem dadurch definierten ,optimalen“ Entnahmeverhältnis $\pi_{\text {opt }}$, das sich mit steigendem $A$ zu kleineren Entnahmeverhältnissen verschiebt.

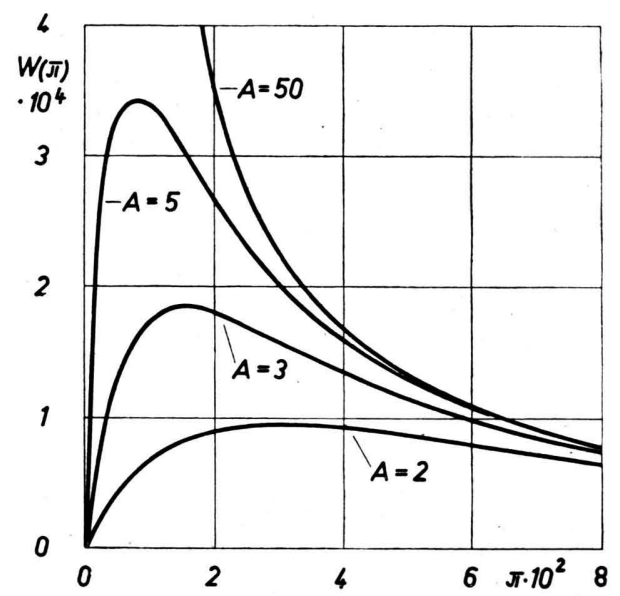

Abb. 1. Die Wertfunktion in Abhängigkeit vom Entnahmeverhältnis $\left(n_{\mathrm{u}}=1,1 \cdot 10^{-2}, \varepsilon=2,6 \cdot 10^{-2}\right.$, vgl. $\left.{ }^{18}\right)$.

Die Funktion $\pi_{\text {opt }}(A)$ ist in Abb. 2 wiedergegeben. Entnimmt man daraus einige Wertepaare $A, \pi_{\text {opt }}$ und berechnet mit ihnen auf Grund von (6) die dazugehörigen Werte $a=a\left(\pi_{\mathrm{opt}}\right)$ (vgl. Tab. 1), so bemerkt man, daß sich die Optimalbedingung für $\pi$ in weiten Grenzen durch

$$
a\left(\pi_{\mathrm{opt}}\right)-1 \approx \frac{1}{2}(A-1)
$$

bzw. $\quad a\left(\pi_{\text {opt }}\right) \approx \frac{1}{2}(A+1)$

formulieren läßt. Um die durch $p$ und $a$ vorgegebene Trennaufgabe mit optimalem Entnahmeverhältnis zu lösen, hat man also die Kolonnenlänge so zu wählen, daß der Anreicherungsfaktor ohne Entnahme

$$
A \approx 2 a-1
$$

beträgt. Die erforderliche Blausäurestromstärke $l$ ergibt sich dann mit Hilfe von Abb. 2 aus dem zu $A=2 a-1$ gehörigen optimalen Entnahmeverhältnis $\pi_{\mathrm{opt}}$ in der Form ${ }^{8}$

$$
l=\frac{p}{\pi_{\mathrm{opt}}} .
$$

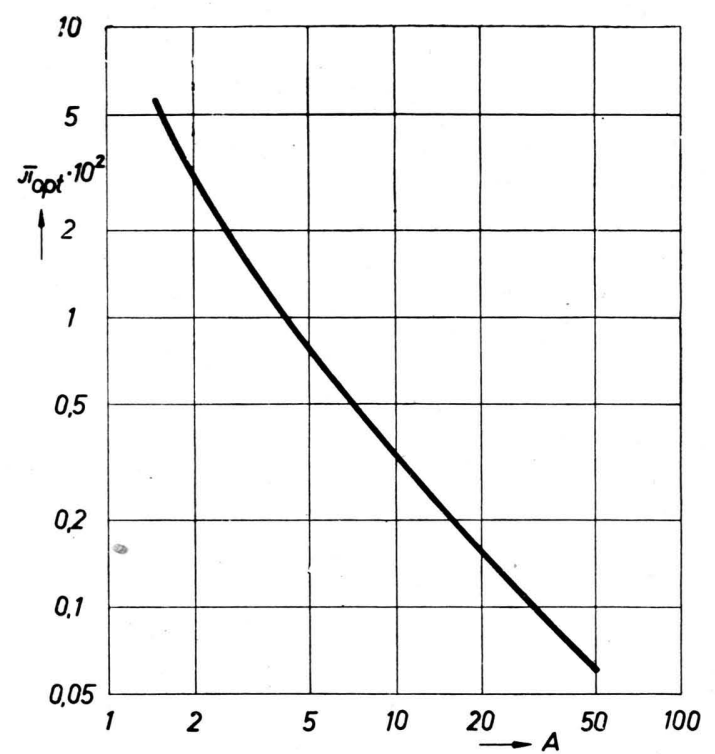

Abb. 2. Das optimale Entnahmeverhältnis in Abhängigkeit vom Anreicherungsfaktor ohne Entnahme, $A$, für $\varepsilon=2,6 \cdot 10^{-2}$ und $n_{\mathrm{u}}=1,1 \cdot 10^{-2}$.

\begin{tabular}{|c|c|c|c|}
\hline$A$ & $\pi_{\text {opt }} \cdot 10^{2}$ & $a\left(\pi_{\text {cpt }}\right)$ & $\frac{a\left(\pi_{\text {opt }}\right)-1}{A-1}$ \\
\hline 1,5 & 5,65 & 1,28 & $0,55_{5}$ \\
2 & 3,05 & 1,53 & 0,53 \\
3 & 1,60 & 2,04 & 0,52 \\
5 & 0,80 & 3,03 & 0,51 \\
10 & 0,330 & 5,58 & 0,51 \\
20 & 0,160 & 10,28 & 0,49 \\
50 & 0,060 & 24,75 & $0,48_{5}$ \\
\hline
\end{tabular}

Tab. 1. Zur Optimalbedingung für das Entnahmeverhältnis.

\section{Das Trennvermögen und die optimalen} Betriebsbedingungen

Bei der Bestimmung des optimalen Entnahmeverhältnisses mit Hilfe der Wertfunktion wurden die oben erwähnten Betriebsbedingungen der Kolonne als konstant angenommen. Es ergibt sich nun die Frage, unter welchen Betriebsbedingungen der bei gestellter Trennaufgabe durch die Optimalbedingung $(10 \mathrm{c})$ festgelegte Anreicherungsfaktor $A$ und Durchsatz $L$ zweckmäßig realisiert wird. Die Antwort kann offenbar nur auf Grund eines weiteren, auswählenden Gesichtspunktes gegeben werden.

${ }^{8} \mathrm{Da}$ aus Abb. 2 allgemein folgt: $\pi_{\mathrm{opt}} \ll 1$, kann man $l$ und $L$ praktisch gleichsetzen, wovon im folgenden Gebrauch gemacht wird. 
Da der Chemikalienverbrauch und der beim chemischen Austauschverfahren damit gekoppelte Energieaufwand bereits bei der Definition derWertfunktion berücksichtigt wurden, kommt zur Festlegung optimaler Betriebsbedingungen praktisch nur noch der Gesamtinhalt der Kolonne an austauschfähigem Material in Frage, der bei vorgegebenem Wert des Elementareffektes $\varepsilon$ ein Maß für die Einstellzeit ${ }^{9}$ der Kolonne ist. Daher soll von allen Kolonnen, die mit einem Durchsatz $L$ arbeiten und einen Anreicherungsfaktor $A$ erzielen, diejenige ausgezeichnet werden, deren Inhalt an austauschfähiger Substanz

$$
J=Q Z i
$$

am kleinsten ist. ( $Z=$ Rohrlänge, $i=$ spezifischer Kolonneninhalt = Inhalt der Kolonne an austauschfähigem Material pro Volumeneinheit.)

Wegen der komplizierten Verhältnisse in einer Füllkörperkolonne ist es unmöglich, das neue Variationsproblem rein theoretisch zu lösen. Man ist vielmehr gezwungen, den Einfluß der verschiedenen Parameter experimentell zu untersuchen.

Dabei ist es unpraktisch, unter Konstanthaltung von $A$ und $L$ etwa die Rohrlänge und -weite zu variieren. Man untersucht einfacher die Abhängigkeit des Anreicherungsfaktors $A$ und des Kolonneninhaltes $J$ vom Durchsatz und von den anderen Betriebsbedingungen für eine Kolonne mit vorgegebener Länge und Weite.

Deshalb eliminiert man aus (12) die Kolonnenlänge $Z$ mit Hilfe der bekannten, für Rektifikationskolonnen gültigen Formel

$$
A=e^{Z / 4},
$$

in der die ,Trennlänge“ $\Lambda$ die Länge eines Kolonnenstückes mit dem Anreicherungsfaktor $e$ angibt. Außerdem führt man an Stelle von $Q$ die spezifische Kolonnenbelastung $\sigma=L / Q$ ein, wodurch man für den Inhalt der Kolonne an austauschfähiger Sub$\operatorname{stanz}$

$$
J=L \ln A \frac{\Lambda i}{\sigma}
$$

erhält. Da $(L \ln A)$ eine durch die Trennaufgabe und das optimale Entnahmeverhältnis festgelegte Konstante ist, hat man die Versuchsbedingungen so

\footnotetext{
9 'Streng genommen ist die Zeit, die bis zum Erreichen des Anreicherungsfaktors $A$ vergeht, für alle Kolonnen unendlich groß (vgl. Abschn. 4). Man versteht
}

zu wählen, daß der Bruch in (14) zu einem Minimum, die Funktion

$$
U=\frac{\sigma}{\Lambda i}
$$

also zu einem Maximum wird. Da $U$ nur spezifische Kolonnendaten enthält, können die optimalen Versuchsbedingungen auf Grund des Extremalprinzips für $U$ mit einer Kolonne beliebiger Abmessung festgelegt werden.

Wie man aus der Umschreibung von (14)

$$
U=\frac{L \cdot \ln A}{J}
$$

erkennt, wird für eine Kolonne mit vorgegebener Länge und vorgegebenem Querschnitt durch das Maximum von $U$ derjenige Betriebszustand ausgewählt, der einen möglichst großen Anreicherungsfaktor $A$ bei möglichst großem Durchsatz und möglichst kleinem Kolonneninhalt ergibt. Die Funktion $U$ wird daher im folgenden ,Trennvermögen“ genannt.

\section{Der zeitliche Verlauf der Anreicherung}

Definitionsgemä $\beta$ wird durch das Maximum des Trennvermögens derjenige Betriebszustand ausgewählt, bei dem für eine Kolonne mit vorgegebenem $L$ und $A$ der Kolonneninhalt $J$ am kleinsten ist, was bei vorgegebenem Elementareffekt $\varepsilon$ dem Minimum der Einstellzeit entspricht. Die Einstellzeit ist jedoch nur dann ein Maß für die Zeit, die bis zum Erreichen der Produktionskonzentration vergeht (Anlaufzeit), wenn sich die Betrachtung auf ein bestimmtes (etwa das optimale) Entnahmeverhältnis bezieht. Da der Zeitfaktor bei der Festlegung des optimalen Entnahmeverhältnisses nicht berücksichtigt wurde, soll nachträglich untersucht werden, inwieweit das aus dem Maximum der Wertfunktion bestimmte Entnahmeverhältnis dem Wunsch nach kurzer Anlaufzeit entspricht. Dabei setzen wir voraus, daß der Anlage während der Anlaufzeit nichts entnommen wird.

Für den zeitlichen Verlauf des Anreicherungsfaktors gilt nach Cohen $^{3 \mathrm{a}}$

$$
a(t)=A-c_{1} e^{-\left|p_{1}\right| t}-c_{2} e^{-\left|p_{2}\right| t}-\ldots
$$

Da die vollständige Gl. (17) für die weitere Rechnung zu unhandlich ist, beschränken wir uns wegen

daher unter „Einstellzeit“ eine praktische Einstellzeit, nach der an der Endanreicherung $(A-1)$ noch ein bestimmter Bruchteil fehlt. 
$c_{1} \gg c_{2}>\ldots$ und $\left|p_{1}\right| \ll\left|p_{2}\right|<\ldots$ auf die beiden ersten Glieder. Dann wird $1 /\left|p_{1}\right|$ gleich der Relaxationszeit $t_{\mathrm{r}}{ }^{10}$, während $c_{1}$ wegen der Anfangsbedingung $a(0)=1$ gleich $A-1$ gesetzt werden muß. Es verbleibt

$$
a(t)-1=(A-1)\left[1-e^{-t / t_{r}}\right] .
$$

Für die Relaxationszeit ergibt $\operatorname{sich}^{3 a}$

$$
t_{\mathrm{r}}=\frac{J}{\varepsilon L F(A) \ln A}
$$

oder mit dem von uns verwendeten Trennvermögen $U$ nach Gl. (15)

$$
t_{\mathrm{r}}=\frac{1}{\varepsilon U F(A)} .
$$

Die Funktion $F(A)$, die sich nur numerisch berech; nen läßt, ist in $^{3 \mathrm{a}}$ graphisch dargestellt.

Gl. (20) zeigt, daß die Relaxationszeit und die zu ihr proportionale Einstellzeit für eine Kolonne mit vorgegebenem $A$ nur durch das Trennvermögen $U$ und den Elementareffekt $\varepsilon$ bestimmt ist. Bei einer Variation der Betriebsbedingungen, bei der sich $\varepsilon$ ändert, ist das Minimum der Einstellzeit also nicht durch das Maximum von $U$, sondern von $\varepsilon U$ gegeben.

Zum Beweis für die Brauchbarkeit der vereinfachten Formel (18) ist in Abb. 3 der experimentell bestimmte Verlauf von $a(t)$ mit dem nach (18) berechneten verglichen. Für die theoretische Kurve wurden $A$ und $t_{\mathrm{r}}$ aus dem Experiment übernommen. Die Meßpunkte liegen im gesamten Bereich innerhalb der Fehlergrenze auf der nach Gl. (18) berechneten Kurve.

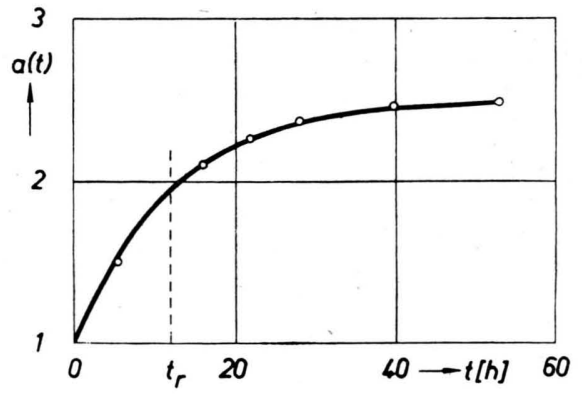

Abb. 3. Die Zeitabhängigkeit des Anreicherungsfaktors. Die Kreise sind Meßpunkte, die eingezeichnete Kurve gibt den theoretischen Verlauf nach Gl. (18).

10 Nach der Relaxationszeit $t_{\mathrm{r}}$ fehlt an der Endanreicherung $A-1$ noch der Bruchteil 1/e, d. h.

$$
a\left(t_{\mathrm{r}}\right)-1=\left(1-\frac{1}{e}\right)(A-1) .
$$

Wir stellen nun die Frage, wie die Anlaufzeit $t_{\mathrm{p}}$, die bis zum Erreichen eines vorgegebenen Anreicherungsfaktors $a_{\mathrm{p}}$ vergeht, vom Entnahmeverhältnis $\pi$ und damit von $A$ abhängt. Aus (18) folgt zunächst $\operatorname{mit} a\left(t_{\mathrm{p}}\right)=a_{\mathrm{p}}{ }^{11}$

$$
t_{\mathrm{p}}=\frac{1}{\varepsilon U F(A)} \ln \frac{A-1}{A-a_{\mathrm{p}}} .
$$

Durch Umformen von Gl. (6) findet man den $\mathrm{Zu}$ sammenhang

$$
A=\left[\frac{(1-\pi) \varepsilon a}{\varepsilon-\pi(a-1+\varepsilon)}\right]^{1 /\left(1+\frac{\alpha \pi}{\varepsilon}\right)},
$$

der, mit $a=a_{\mathrm{p}}$ in (21) eingesetzt, die gesuchte Beziehung zwischen $t_{\mathrm{p}}$ und $\pi$ liefert. Sie ist in Abb. 4 für einige Werte von $a_{\mathrm{p}}$ graphisch dargestellt. Demnach zeigt $t_{\mathrm{p}}$ ein Minimum bei einem Entnahmeverhältnis, das für kleine $a$ nahezu bei $\pi_{\text {opt }}$ liegt. Die Abweichungen, die sich bei größeren $a$-Werten ergeben, sind praktisch bedeutungslos, da die Minima der Kurven relativ flach verlaufen. Daraus folgt, daß eine Entnahme mit $\pi=\pi_{\text {opt }}$ auch hinsichtlich der Anlaufzeit besonders günstig ist.

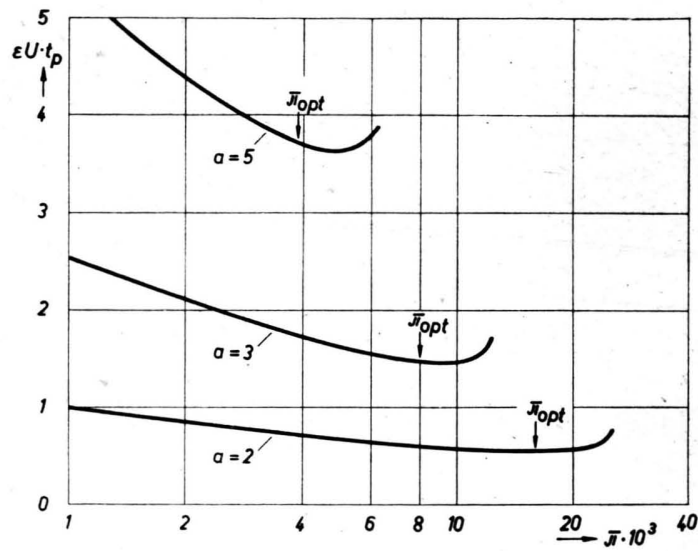

Abb. 4. Die Anlaufzeit $t_{\mathrm{p}}$ bis zum Erreichen verschiedener Anreicherungsfaktoren $a$ in Abhängigkeit vom Entnahmeverhältnis $\left(n_{\mathrm{u}}=1,1 \cdot 10^{-2}, \varepsilon=2,6 \cdot 10^{-2}\right)$.

5. Die experimentelle Bestimmung der optimalen Betriebsbedingungen und des effektiven Elementareffektes

Zur Festlegung der optimalen Betriebsbedingungen wurden mit einer 2,6 m langen Kolonne 60 Entmischungsversuche durchgeführt, wobei ein geringfügiger ,Wandeffekt" (s. u.) durch Verwendung von

${ }^{11}$ Aus (21) folgt, da $B$ bei optimalem Betrieb einer Kolonne $\left[a_{\mathrm{p}}-1=1 / 2(A-1)\right]$ die Anlaufzeit $t_{\mathrm{p}}=$ $t_{\mathrm{r}} \ln 2$ beträgt. 
zwei verschiedenen Kolonnenweiten (14,4 und 24,8 mm) erfaßt werden konnte. Die Art der Blausäureerzeugung, der Flüssigkeitsbewegung und der Gassteuerung sowie weitere betriebstechnische Einzelheiten sind in der folgenden Arbeit beschrieben und sollen daher hier übergangen werden.

Im stationären Endzustand wurden aus der Kolonne jeweils am oberen und unteren Ende etwa $0,5 \mathrm{~cm}^{3}$ Flüssigkeit entnommen und auf dem Umweg über $\mathrm{CO}$ in $\mathrm{CO}_{2}$ verwandelt ${ }^{12}$. Die $\mathrm{CO}_{2}$-Proben konnten in einem Massenspektrometer ${ }^{13}$ mit einer relativen Genauigkeit von $\pm 0,5 \%$ auf ihren ${ }^{13} \mathrm{C}$-Gehalt untersucht werden. Durch Entnahme weiterer Proben in angemessenen Zeitabständen wurde stets nachgeprüft, ob der stationäre Endzustand auch wirklich vorlag. Die Natronlauge enthielt als Stabilisator zur Verminderung der Blausäurepolymerisation $0,2 \mathrm{Mol} \mathrm{Na}{ }_{2} \mathrm{SO}_{3}$ pro Liter.

Es war natürlich nicht möglich, das Trennvermögen für alle Kombinationen der in Frage kommenden Parameterwerte zu untersuchen. So wurde der Einfluß des Gasdrucks, der Natronlaugekonzentration und der Füllkörperform nur durch orientierende Versuchsreihen bestimmt, was völlig ausreichend war, da diese Größen durch sekundäre Gesichtspunkte ohnehin weitgehend festgelegt sind.

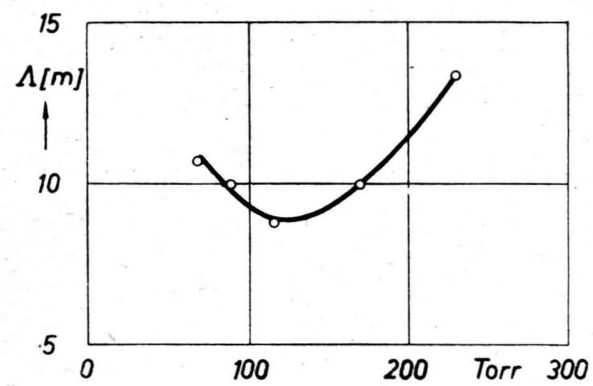

Abb. 5. Die Trennlänge $A$ in Abhängigkeit von dem am unteren Ende der Kolonne gemessenen Gasdruck. $(Z=2,5 \mathrm{~m}$, Kolonnenweite $=24,8 \mathrm{~mm}$, Natronlaugedurchsatz $=645 \mathrm{~cm}^{3} / \mathrm{h}$ einer 4,5- $n$. Lösung.) Der Staudruck längs der Kolonne nahm im untersuchten Bereich mit steigendem Gesamtdruck ungefähr linear von 11 auf 7 Torr ab.

a) Abb. 5 zeigt die Druckabhängigkeit der Trennlänge für die weite, mit Glaswendeln gefüllte Kolonne bei konstantem Durchsatz. Die Trennlänge $\Lambda$ besitzt demnach ein deutliches Minimum, die

12 E. W. Becker u. W. Vogell, Z. Physik 130, 129 [1951].

13 E.W. Becker, E. Dörnenburgu. W. Walcher, Z. angew. Physik 2, 261 [1950].

14 Die Konzentration der gelösten Blausäure beträgt
Trennschärfe $\mathbf{l} / \Lambda$ also ein Maximum bei einem Gasdruck von etwa 125 Torr. Der Anstieg der Trennlänge unterhalb von 125 Torr dürfte im wesentlichen auf den mit fallendem Gesamtdruck prozentual anwachsenden Fremdgasdruck (Wasserdampf, Luftspuren) zurückzuführen sein, während der Anstieg oberhalb des Minimums wohl hauptsächlich auf der Druckabhängigkeit der elementaren Isotopenverschiebung zwischen Gas und Flüssigkeit beruht. Der letzte Effekt ist eine Folge der in der Flüssigkeit gelösten Blausäure ${ }^{14}$, deren Austauschreaktion

$$
\begin{aligned}
\left(\mathrm{H}^{13} \mathrm{CN}\right)_{\text {gas }}+\left(\mathrm{H}^{12} \mathrm{CN}\right)_{\text {gelöst }} & \rightleftharpoons\left(\mathrm{H}^{12} \mathrm{CN}\right)_{\text {gas }} \\
& +\left(\mathrm{H}^{13} \mathrm{CN}\right)_{\text {gelöst }}
\end{aligned}
$$

zu einer umgekehrten Isotopenverschiebung führt wie die Austauschreaktion (1) der gelösten Cyanidionen ${ }^{1,3 a}$. Bei allen weiteren Versuchen wurde der Anfangsdruck im Hinblick auf die starke Polymerisationsneigung der gelösten Blausäure mit 115 Torr etwas niedriger gewählt als der aus Abb. 5 abgeleitete Optimaldruck.

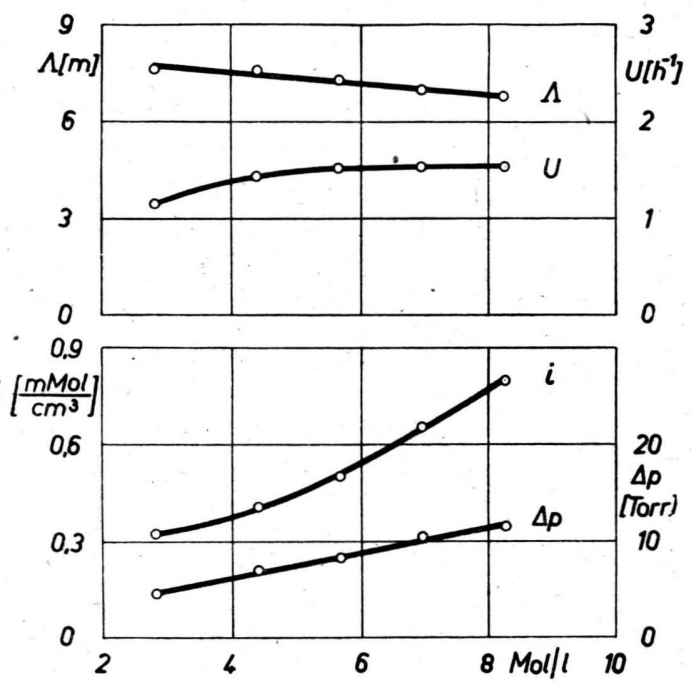

Abb. 6. Der Einfluß der Natronlaugekonzentration und damit der CN-Ionenkonzentration auf die Trennlänge $\Lambda$, den spezifischen Kolonneninhalt $i$, das Trennvermögen $U$ und den Staudruck $\Delta p$ für eine Förderleistung der Natronlaugepumpe von $165 \mathrm{~cm}^{3} / \mathrm{h}$ (Kolonnenweite $=14,4 \mathrm{~mm}$ ).

b) Der Einfluß der Natronlaugekonzentration auf das Trennvermögen geht aus der in Abb. 6 dargestellten Verșuchsreihe hervor. Die Trennlänge $\Lambda$

bei einem mittleren Partialdruck von 100 Torr ungefähr 0,6 Mol/Ltr.; das sind bei einer $\mathrm{NaOH}-K o n z e n-$ tration von 4,5 Mol/Ltr. etwa $12 \%$ des Gesamtinhaltes der Flüssigkeit an austauschfähigem Material. 
nimmt mit steigender Ionenkonzentration der Lösung schwach ab, was hauptsächlich auf dem relativ geringer werdenden Einfluß des Austauschgleichgewichtes (22) beruhen dürfte. Da der spezifische Kolonneninhalt $i$ jedoch mit steigender $\mathrm{NaOH}-\mathrm{Kon}$ zentration stark zunimmt, wird das in Abb. 6 ebenfalls eingezeichnete Trennvermögen $U$ oberhalb von etwa $5 \mathrm{Mol} \mathrm{NaOH} / \mathrm{Ltr}$. nahezu konstant, obwohl die im Ausdruck für $U$ im Zähler stehende spezifische Kolonnenbelastung mit der Natronlaugekonzentration anwächst. Da nach dem oben Gesagten der effektive Elementareffekt $\varepsilon$ mit steigender Cyanidionenkonzentration zunimmt und die Relaxationszeit nach (20) umgekehrt proportional zu $\varepsilon U$ ist, wird man im Interesse einer kleinen Einstellzeit die Natronlaugekonzentration möglichst groß wählen. Wir haben uns bei den folgenden Versuchen jedoch auf 4,5 Mol NaOH/Ltr. beschränkt, da sich diese Konzentration in einer längeren Kaskade aus sekundären Gründen nicht wesentlich überschreiten läßt. (Vgl. Abschnitt B 3 sowie die folgende Arbeit.)

c) Die Variationsmöglichkeiten der Füllkörperform sind relativ gering, da als Füllkörpermaterial wegen der Aggressivität der Lösungen praktisch nur Glas oder Keramik in.Frage kommen. Von den handelsüblichen Füllkörpersorten wurden $5 \mathrm{~mm}$-BerlSättel aus Porzellan und $4 \mathrm{~mm}$-Glaswendeln ${ }^{15}$ untersucht. Sie zeigten hinsichtlich des Trennvermögens sehr ähnliche Ergebnisse, doch war der Strömungswiderstand der Berlsättelpackung um $60-80 \%$ größer als der der Glaswendelfüllung. Aus diesem Grund und weil die Porzellansättel infolge ihrer geringen Elastizität zuweilen die Glasrohre sprengten, wurden für die weiteren Versuche ausschließlich Glaswendeln benutzt.

d) In der Rektifikationstechnik ist es üblich, die Füllkörpergröße dem Rohrdurchmesser anzupassen, indem man das Verhältnis von Rohr- zu Füllkörperdurchmesser etwa gleich 10 wählt. Nach unseren Versuchen ließen sich jedoch wesentlich kleinere Füllkörper als solche mit $4 \mathrm{~mm} \varnothing$ auch in den engeren Kolonnen nicht verwenden, da sie bei den relativ großen Gasgeschwindigkeiten ein Hochwirbeln der Flüssigkeit verursachen. Es wurden daher alle Kolonnenweiten einheitlich mit $4 \mathrm{~mm}$-Glaswendeln beschickt.

15 Die Glaswendeln bestehen aus $4-5$ Windungen eines ungefähr $1 \mathrm{~mm}$ starken Glasfadens.

${ }^{16}$ Wegen der gelösten Blausäure ist der Gasdurchsatz $l$ sowie der Flüssigkeitsdurchsatz $L$ an austausch- e) Besondere Aufmerksamkeit haben wir der Oberflächenbeschaffenheit der Füllkörper zugewendet. Es hatte sich nämlich gezeigt, daß die Wirksamkeit der Kolonnenfüllung durch eine geeignete Vorbehandlung erheblich gesteigert werden kann. Zur „Aktivierung" wurden die durch Klopfen weitgehend sedimentierten Füllkörper zunächst mehrmals mit der wäßrigen Lösung eines technischen Benetzungsmittels behandelt. Die Kolonne wurde sodann bei 115 Torr in Betrieb genommen und, nachdem sie vollständig mit Blausäure gefüllt war, nochmals mit verdünnter Natronlauge übergossen. Die Natronlauge dringt dabei unter Absorption der Blausäure in alle Zwischenräume ein und bewirkt eine intensive, lange Zeit haltbare Benetzung der Füllkörperoberfläche. Der außerordentliche Erfolg dieser Methode geht aus Abb. 7 hervor, die den Anreicherungsfaktor der weiten Kolonne vor und nach der Aktivierung in Abhängigkeit von der Natronlaugestromstärke zeigt ${ }^{16}$. Die Wirkung ist besonders stark bei kleiner spezifischer Kolonnenbelastung, wo die Größe $A-1$ durch die Aktivierung um den Faktor 7 gesteigert werden konnte. Der Kolonneninhalt wird durch die Aktivierung praktisch nicht beeinflußt. Die Haltbarkeit des aktivierten Zustandes wurde in einem fünftägigen Dauerversuch kontrolliert. Während dieser Zeit war kein Abfall der Trennschärfe zu beobachten.

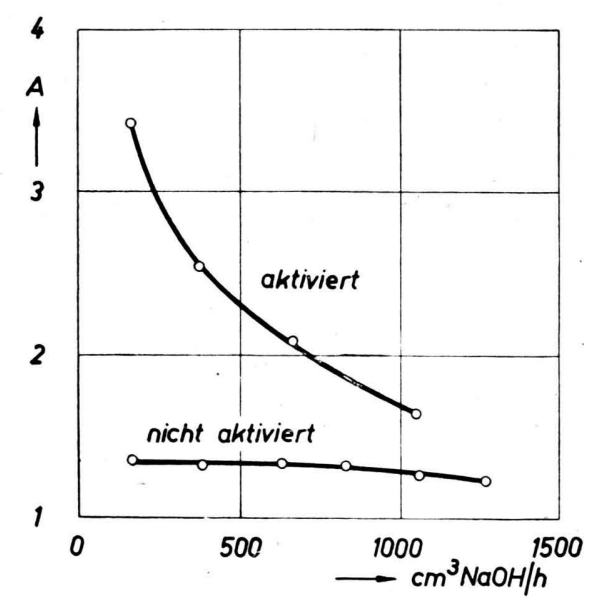

Abb. 7. Der Anreicherungsfaktor der weiten Kolonne in Abhängigkeit von der Förderleistung der Natronlaugepumpe für aktivierte und nicht aktivierte Füllkörper.

fähigem Material $\left(\mathrm{CN}^{\prime}\right.$ und $\left.\mathrm{HCN}_{\text {gelöst }}\right)$ um ungefähr $12 \%$ größer als die in Abb. 7,8 u. 9 angegebenen $\mathrm{NaOH}-$ Stromstärken. 
f) Auf Grund der bei der Füllkörperaktivierung gemachten Erfahrungen ergab sich die Frage, ob man der Natronlauge zur Verbesserung der Benetzungsverhältnisse während des Betriebes ein Netzmittel zusetzen kann. Gegen diese Arbeitsweise bestanden zunächst Bedenken, da sich Netzmittel als oberflächenaktive Stoffe bekanntlich in der Flüssigkeitsoberfläche stark anreichern und dadurch den Gasaustausch zwischen den Phasen möglicherweise behindern können. Es zeigte sich jedoch, daß Zusätze von Fewa oder Octylalkohol, die die Oberflächenspannung der Cyanidlösung um $30-50 \%$ erniedrigten, die Trenneigenschaften der Kolonne nicht merklich beeinflussen. Es muß daraus geschlossen werden, daß die geschwindigkeitsbestimmenden Austauschvorgänge im Gas oder in der Flüssigkeit selber, nicht aber an der Flüssigkeitsoberfläche passieren. Die weiter beobachtete Tatsache, daß durch eineVerminderung der Oberflächenspannung der Lösung keine merkliche Verkleinerung des spezifischen Kolonneninhaltes zu erzielen ist, deutet darauf hin, daß der spezifische Kolonneninhalt nicht wesentlich von der Oberflächenspannung, sondern von der Zähigkeit der Lösung abhängt.

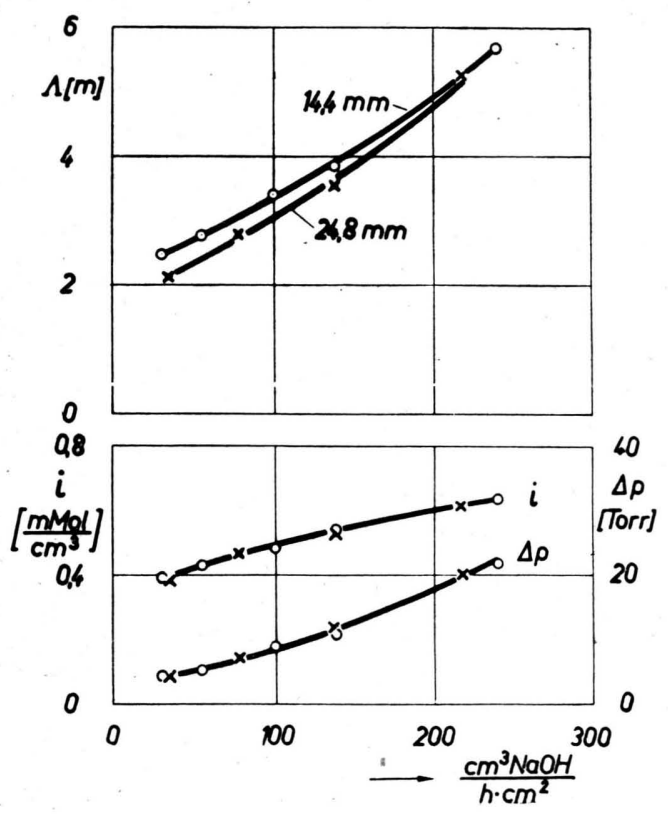

Abb. 8. Die Trennlänge $A$, der spezifische Kolonneninhalt $i$ und der Staudruck $\Delta p$ in Abhängigkeit von der spezifischen Kolonnenbelastung bei konstanter $\mathrm{NaOH}-K o n z e n t r a t i o n ~(4,5 \mathrm{Mol} / \mathrm{Ltr}$.) für die aktivierte enge und weite Kolonne (o bzw. $\times$ ). g) In Abb. 8 sind die Trennlänge, der spezifische Kolonneninhalt und der Staudruck in Abhängigkeit von der spezifischen Kolonnenbelastung ${ }^{16}$ für die aktivierte enge und weite Kolonne wiedergegeben. Abb. 9 zeigt den Verlauf des daraus berechneten

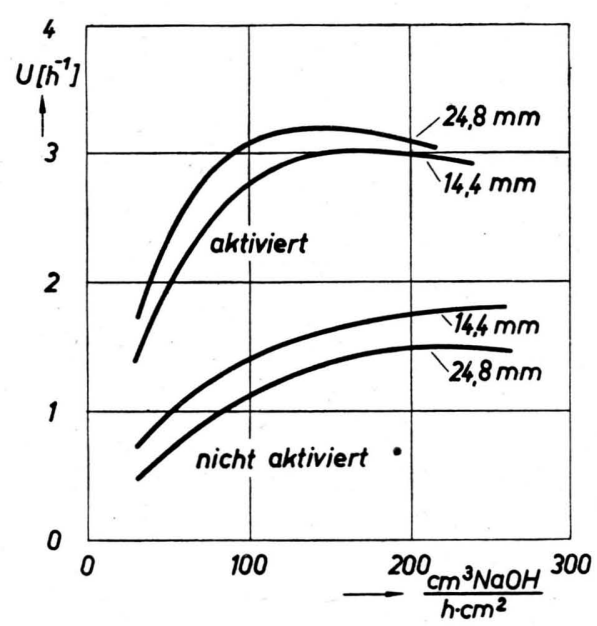

Abb. 9. Das aus den experimentellen Kurven der Abb. 8 berechnete Trennvermögen $U$ der aktivierten Kolonnen in Abhängigkeit von der spezifischen Kolonnenbelastung. Zum Vergleich ist auch das Trennvermögen der nicht aktivierten Kolonnen eingezeichnet.

Trennvermögens zusammen mit dem Trennvermögen der beiden Kolonnen vor der Aktivierung. Das Trennvermögen wird demnach durch die Aktivierung um einen Faktor 2-3,5 gesteigert. Dabei ist die Aktivierung besonders stark bei kleinen spezifischen Kolonnenbelastungen wirksam. Während die weite Kolonne im nicht aktivierten Zustand durchweg ein etwas kleineres Trennvermögen besitzt als die enge, liegen die Verhältnisse im aktivierten $\mathrm{Zu}$ stand umgekehrt. Dies läßt darauf schließen, daß das kleinere Trennvermögen der nicht aktivierten Kolonnen auf einer Ungleichmäßigkeit der Benetzung (,Bachbildung“) beruht, die in einer engen Kolonne erfahrungsgemä $ß$ geringer ist als in einer weiten. Bei den aktivierten Kolonnen, bei denen die Benetzung wesentlich gleichmäßiger sein dürfte, macht sich die Begrenzung der Kolonnenfüllung durch die Rohrwand störend bemerkbar. Dieser „Wandeffekt" fällt natürlich bei der weiten Kolonne wegen ihres günstigeren Verhältnisses von Rohrquerschnitt zu -umfang weniger ins Gewicht. Die Trennvermögen der aktivierten Kolonnen besitzen beide ein Maximum bei einer spezifischen Kolonnenbelastung von etwa $160 \mathrm{~cm}^{3} \mathrm{NaOH} / \mathrm{h} \cdot \mathrm{cm}^{2}$. 
h) Die experimentelle Bestimmung des effektiven Elementareffektes: Die gute Übereinstimmung der Gl. (18) mit dem gemessenen zeitlichen Verlauf des Anreicherungsfaktors (vgl. Abb. 3) legt es nahe, aus der experimentell bestimmten Relaxationszeit mit Hilfe von (19) die Größe $\varepsilon$ zu berechnen. Die Hauptschwierigkeit besteht dabei in der genauen Bestimmung des Kolonneninhaltes $J$, so daß die erreichbare Fehlergrenze für $\varepsilon$ im wesentlichen von der für $J$ abhängt.

In Tab. 2 sind fünf Versuche ausgewertet, die z.T. mit der engen und z. T. mit der weiten Kolonne durchgeführt wurden. Für $\varepsilon$ ergibt sich ein Mittelwert von ${ }^{17}$

$$
\varepsilon=0,025 \pm 0,0025,
$$

der mit der Literaturangabe ${ }^{18}$ innerhalb der Fehlergrenze übereinstimmt.

\begin{tabular}{|c|c|c|c|c|c|c|}
\hline $\begin{array}{c}\text { Vers. } \\
\text { Nr. }\end{array}$ & $\begin{array}{c}L \\
{[\mathrm{Mol} / \mathrm{h}]}\end{array}$ & $A$ & $F(A)$ & $\begin{array}{c}t_{\mathrm{r}} \\
{[\mathrm{h}]}\end{array}$ & $\begin{array}{c}J \\
{[\mathrm{Mol}]}\end{array}$ & $\varepsilon \cdot 10^{2}$ \\
\hline 1 & 0,44 & 2,50 & 1,95 & 12,0 & $0,26_{5}$ & 2,82 \\
2 & $0,24_{5}$ & 2,90 & 1,35 & 28,5 & 0,23 & 2,30 \\
3 & $1,80_{5}$ & 2,54 & 1,85 & 9,5 & $0,77_{5}$ & 2,62 \\
4 & $0,80_{5}$ & 3,45 & 0,94 & 26,0 & $0,58_{5}$ & 2,40 \\
5 & 0,44 & 2,78 & $1,50$. & 14,5 & 0,23 & 2,36 \\
\hline
\end{tabular}

Tab. 2. Zur Bestimmung des effektiven Elementareffektes.

\section{B. Die Trennkaskade}

\section{Das Staffelungsprinzip}

Will man mit einer Trennanlage einen Anreicherungsfaktor $a \gtrsim 3$ erzielen, so ist es zweckmäßig, die Trennanlage entsprechend Abb. $10 \mathrm{zu}$ staffeln. Man betreibt dann eine weite Kolonne $R_{1}$ mit dem Blausäurestrom $l_{1}$, von dem am Kopf der Kolonne nur der Stromanteil $l_{1}-l_{2}$ durch Zugabe von $\mathrm{NaOH}$ in die flüssige Phase übergeführt wird. Von dem verbleibenden Blausäurestrom $l_{2}$, der eine engere Kolonne $R_{2}$ speist, wird an deren oberem Ende nur der Anteil $l_{2}--l_{3}$ von der zugegebenen $\mathrm{NaOH}$ aufgenommen usw. In Abb. 10 ist als Beispiel das Schema einer vierstufigen Kaskade wiedergegeben, doch kann die Stufenzahl natürlich auch größer oder kleiner sein.

Die Möglichkeit, den Kolonnendurchsatz und damit die Kolonnenweite mit fortschreitender Anreicherung des seltenen Isostops zu vermindern, folgt aus der Tatsache, daß der maximal mögliche Isotopentransport durch einen bestimmten Kolonnenquerschnitt mit dem Molenbruch des anzureichernden Isotops anwächst.

17 Dieser Wert dés effektiven Elementareffektes gilt für $T=20^{\circ} \mathrm{C}$, eine Konzentration von $4,5 \mathrm{Mol} / \mathrm{Ltr}$. der auf die Kolonne gegebenen Natronlauge und einen mittleren Gasdruck von ungefähr 110 Torr.

${ }^{18}$ H. C. Urey, J. chem. Soc. [London] 1947, 562.
Da am oberen Ende einer Kaskade jedoch nur der Transport entnommen werden kann, der durch die Basis hindurchtritt, läßt sich der große Transport in den oberen Teilen einer Anlage mit konstantem Durchsatz nichtvollausnutzen. Er wird daher durch Verminderung des Blausäuredurchsatzes unter entsprechender Verengung der Rohre zweckmäßig herabgesetzt, woraus sich eine erheblicheVerkleinerung des Inhaltes und damit eine Verkürzung der Anlaufzeit ergibt.

Man vermutet bereits, daß eine nahezu kontinuierlich gestaffelte Anlage, die aus möglichst vielen, sehr kurzen Einzelkolonnen

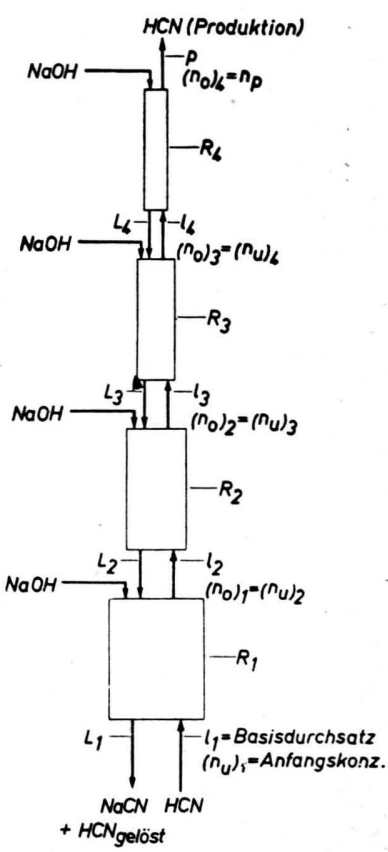

Abb. 10. Das Schema einer aus vier Stufen bestehenden Austausch-Kaskade. besteht, hinsichtlich der Anlaufzeit die günstigsten Resultate liefert. Da jede Staffelstelle jedoch eine zusätzliche Natronlaugepumpe erfordert ${ }^{19}$, und die Anlaufzeit bei mehrfacher Unterteilung der Anlage mit steigender Stufenzahl nur noch wenig sinkt (s. u.), wird man im allgemeinen so staffeln, daß sich pro Stufe noch ein Anreicherungsfaktor von $2-3$ ergibt. Die Stufenlänge liegt dann in der Größenordnung von einigen Metern, was für die Aufstellung der Anlage in einem Raum normaler Höhe von Vorteil ist.

Géht man davon aus, daß die Länge der Unterabschnitte aus den erwähnten Gründen festliegt, so sind ihre Anreicherungsfaktoren $A$ (ohne Entnahme) bestimmt, wenn man die Betriebsbedingungen und damit $\Lambda$ wie bei der einfachen Trennkolonne mit Hilfe des Trennvermögens auswählt. Bei der Berechnung einer optimalen Kaskade verbleibt also nur die Frage, wieviele der Kolonnen gegebener Länge zur Lösung der gestellten Trennaufgabe erforderlich sind und welchen Durchsatz bzw. Querschnitt die einzelnen Stufen besitzen müssen.

${ }^{19}$ Bei der ${ }^{15} \mathrm{~N}$ - und ${ }^{34} \mathrm{~S}$-Anreicherung erfordert jede Staffelstelle außerdem noch ein Auskochaggregat, in dem die Umwandlung der flüssigen Phase in die Gasphase erfolgt. 
Für die letzte Stufe $\left(R_{4}\right.$ in Abb. 10) gestaltet sich damit das Problem wie für die Einzelkolonne: Aus Abb. 2 entnimmt man das zu dem Anreicherungsfaktor $A$ dieser Stufe gehörige optimale Entnahmeverhältnis, das seinerseits bei gegebenem Entnahmestrom $p$ den Durchsatz und damit den Querschnitt dieser Stufe bestimmt. Der Anreicherungsfaktor $a$, der sich unter der Wirkung des Entnahmestromes $p$ einstellt, folgt aus der Bedingung (10 b).

Für alle übrigen Stufen liegen die Verhältnisse insofern komplizierter, als an ihrem oberen Ende ein Isotopentransport nicht nur auf Grund des Materialstromes $p$ stattfindet, sondern auch dadurch, daß z. B. der aus $R_{3}$ in Richtung $R_{4}$ abfließende Gasstrom eine etwas höhere ${ }^{13} \mathrm{C}$-Konzentration besitzt als die von $R_{4}$ an $R_{3}$ zurückgelieferte Flüssigkeit. Diese „Entnahme zweiter Art" hat auf den Anreicherungsfaktor eine etwas andere Wirkung als die bisher allein behandelte Materialentnahme, für die Gl. (6) zuständig ist. Zur Festlegung einer ,,optimalen Entnahmebedingung" für Kolonnen mit „kombinierter" Entnahme haben wir daher Gl. (6) zu verallgemeinern, was im folgenden geschehen soll.

\section{Die Wertfunktion bei kombinierter Entnahme}

Um das Verhalten des Anreicherungsfaktors im Fall der kombinierten Entnahme zu ermitteln, hat man das Differentialgleichungs-System der Einzelkolonne mit neuen Randbedingungen zu lösen. Nach Cohen ${ }^{3 a}$ gilt für den hier allein interessierenden stationären Zustand bei Vernachlässigung von Gliedern der Größenordnung $\varepsilon n$ gegen $1^{20}$

$$
\begin{aligned}
l \frac{\mathrm{d} r}{\mathrm{~d} z}+k c C(R-\alpha r) & =0, \\
L \frac{\mathrm{d} R}{\mathrm{~d} z}+k c C(R-\alpha r) & =0 .
\end{aligned}
$$

Darin bedeutet $z$ die Koordinate in Rohrrichtung, $r(z)=\frac{n(z)}{1-n(z)}$ das Mischungsverhältnis, $n(z)$ den Molenbruch des ${ }^{13} \mathrm{C}, l$ die Stromstärke und $c$ die Konzentration der austauschfähigen Substanz. Die kleinen Buchstaben gelten für die Gasphase, die großen für die Flüssigkeit. $k$ ist eine Konstante, die pauschal alle die Austauschgeschwindigkeit bestimmenden Vorgänge beschreibt und demnach von den speziellen Betriebsbedingungen abhängt.

${ }^{20}$ Wegen dieser Vernachlässigung sind die aus (23) abgeleiteten Formeln nicht für beliebig große Anreicherungsfaktoren gültig; nach $^{3}$ a ist die angegebene
Bedeutet $z=0$ das obere und $z=Z$ das untere Ende der Kolonne, so lauten die Randbedingungen, die zu Gl. (6) geführt haben

$$
\begin{aligned}
r(Z) & =\text { const. }=\varrho, \\
R(0) & =r(0), \\
L & =l-p .
\end{aligned}
$$

Sie beschreiben den Fall der reinen Materialentnahme. Beim Übergang zur kombinierten Entnahme wird nur Bedingung (25) geändert: Da die Cyanidlösung, die von der nächst höheren Kolonne zurückgeliefert wird, einen etwas kleineren ${ }^{13} \mathrm{C}$-Gehalt besitzt als die von der betrachteten Kolonne abgegebene Blausäure, gilt

mit $\delta \leqq \varepsilon$.

$$
R(0)=(1-\delta) r(0)
$$

Die Lösung des Simultansystems (23) mit den Randbedingungen (24), (25a) und (26) lautet:

$$
\begin{aligned}
& r(z)=\varrho \frac{(1-\pi)(\varepsilon-\delta) e^{-\mu z}+(\pi+\delta)}{(1-\pi)(\varepsilon-\delta) e^{-\mu Z}+(\pi+\delta)}, \\
& R(z)=\varrho \frac{(\varepsilon-\delta) e^{-\mu z}+\alpha(\pi+\delta)}{(1-\pi)(\varepsilon-\delta) e^{-\mu Z}+(\pi+\delta)}
\end{aligned}
$$

mit

$$
\mu=\frac{k c C}{L}[1-\alpha(1-\pi)] .
$$

Aus (27a) erhält man den Anreicherungsfaktor bei kombinierter Entnahme

$a(\pi, \delta)=\frac{r(0)}{\varrho}=\frac{(1-\pi)(\varepsilon-\delta)+(\pi+\delta)}{(1-\pi)(\varepsilon-\delta) e^{-\mu Z}+(\pi+\delta)}$.

Die Bedeutung von $e^{-\mu Z}$ erkennt man, wenn man in (28) $\pi=\delta=0$ setzt: man findet

also

$$
a(0,0)=A=e^{\frac{k c C \varepsilon Z}{L}},
$$

$$
e^{-\mu Z}=A^{-\left(1+\frac{\alpha \pi}{\varepsilon}\right)}
$$

Damit folgt als Endresultat an Stelle von (6)

$$
a(\pi, \delta)=\frac{(1-\pi)(\varepsilon-\delta)+(\pi+\delta)}{(1-\pi)(\varepsilon-\delta) A^{-\left(1+\frac{\alpha \pi}{\varepsilon}\right)}+(\pi+\delta)} .
$$

Um zu der Optimalbedingung für die kombinierte Entnahme zu kommen, betrachten wir zunächst den Grenzfall der reinen Entnahme zweiter Art. Aus (29) ergibt sich mit $\pi=0$

Näherung bis $\varrho A \lesssim 1 / 2$ brauchbar, das entspricht einer ${ }^{13} \mathrm{C}$-Konzentration von $\approx 30 \%$. 


$$
a(\delta)=\frac{A}{1+(A-1) \frac{\delta}{\varepsilon}} .
$$

Die ,optimale“ Entnahme 2. Art wird, analog zu Abschnitt A 2, definiert durch das Maximum der Wertfunktion

$$
W(\delta)=\frac{\tau(\delta)}{l}(a(\delta)-1) .
$$

Dazu benötigt man noch den Transport $\tau$, den man allgemein in der Form

$$
\begin{aligned}
\tau & =L[n(Z)-N(Z)] \\
& =L n(Z)(1-n(Z))\left[1-\frac{R(Z)}{r(Z)}\right]
\end{aligned}
$$

schreiben kann $\left(\operatorname{vgl} .{ }^{3 a}\right)$. Im Fall reiner Entnahme zweiter Art erhält man aus (27) mit $\pi=0$ und $l=L$ unter Berücksichtigung von (30)

$$
\tau(\delta)=l \delta a(\delta) n_{\mathrm{u}}\left(1-n_{\mathrm{u}}\right) .
$$

Damit folgt für die Wertfunktion

$$
W(\delta)=\delta a(a-1) n_{\mathrm{u}}\left(1-n_{\mathrm{u}}\right),
$$

wobei $a$ durch Gl. (30) zu ersetzen ist.

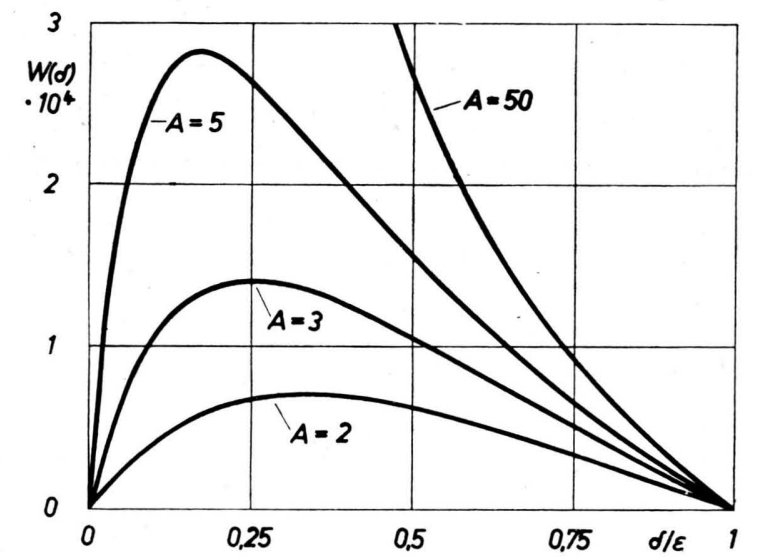

Abb. 11. Die Wertfunktion bei reiner Entnahme zweiter Art in Abhängigkeit von $\delta / \varepsilon$ für $n_{\mathrm{u}}=1,1 \cdot 10^{-2}$.

Der Verlauf von $W(\delta)$ ist in Abb. 11 für einige Parameterwerte $A$ aufgezeichnet. Demnach verschiebt sich das Maximum von $W$ mit steigendem $A$ zu kleineren Werten von $\delta$. Für $\delta=\varepsilon$ wird $W$ bei allen $A$-Werten gleich Null, da die Kolonne dann überhaupt nicht mehr anreichert $[a(\delta=\varepsilon)=1]$. Eine analytische Bestimmung des Maximums von $W$ liefert den allgemeinen Zusammenhang:

$$
\delta_{\text {opt }}=\frac{\varepsilon}{A+1} .
$$

Führt man diesen Optimalwert von $\delta$ in (30) ein, so ergibt sich für den Anreicherungsfaktor der Kolonne bei optimaler Entnahme zweiter Art

$$
a\left(\delta_{\text {opt }}\right)=\frac{1}{2}(A+1),
$$

also die gleiche Bedingung wie bei der reinen Materialentnahme. Man vermutet daher, daß Gl. (36) bzw. (10 b) auch für eine beliebige Kombination der beiden Entnahmearten gültig ist, was sich durch Probieren auch tatsächlich bestätigen läßt. Wir bezeichnen daher allgemein diejenige kombinierte Entnahme als optimal, die den Anreicherungsfaktor $A$ der betrachteten Kolonne auf $(A+1) / 2$ herabsetzt:

$$
a_{\text {opt }}=\frac{1}{2}(A+1) .
$$

\section{Berechnung einer optimalen Kaskade}

Die Berechnung der Kaskade gestaltet sich nun folgendermaßen: Da der Anreicherungsfaktor $A_{\mathrm{s}}$ jeder Stufe durch die vorgesehene Kolonnenlänge und das Maximum des Trennvermögens bestimmt ist, liegen auch die Anreicherungsfaktoren $a_{\mathrm{s}}$ aller Stufen auf Grund der optimalen Entnahmebedingung (37) fest. Die insgesamt erforderliche Stufenzahl $S$ folgt dann aus

$$
\stackrel{S}{\prod_{s=1}} a_{\mathrm{s}}=a_{\mathrm{p}} .
$$

$\mathrm{Zu}$ dem Moldurchsatz $L_{\mathrm{s}}$ der $s$-ten Stufe kommt man auf Grund der Tatsache, daß im stationären Zustand der Transport längs der Anlage konstant ist, die kombinierte Entnahme am Kopf jeder Stufe also gleich der Produktion $p n_{\mathrm{p}}$ sein muß. Es gilt demnach

$$
p n_{\mathrm{p}}=p\left(n_{\mathrm{o}}\right)_{\mathrm{s}}+L_{\mathrm{s}}\left[\left(n_{\mathrm{o}}\right)_{\mathrm{s}}-\left(N_{\mathrm{o}}\right)_{\mathrm{s}}\right] .
$$

Formt man $L_{\mathrm{s}}\left[\left(n_{\mathrm{o}}\right)_{\mathrm{s}}-\left(N_{\mathrm{o}}\right)_{\mathrm{s}}\right]$ analog $\mathrm{zu}$ Gl. (32) um, so erhält man wegen $\left[1-\frac{\left(R_{0}\right)_{\mathrm{s}}}{\left(r_{\mathrm{o}}\right)_{\mathrm{s}}}\right]=\delta_{\mathrm{s}}$ nach Gl. (25a) unter Berücksichtigung von $\frac{p}{L_{\mathrm{s}}} \approx \frac{p}{l_{\mathrm{s}}}=\tau_{\mathrm{s}}$ die Beziehung

$$
\delta_{\mathrm{s}}=\pi_{\mathrm{s}} \frac{n_{\mathrm{p}}-\left(n_{\mathrm{o}}\right)_{\mathrm{s}}}{\left(n_{\mathrm{o}}\right)_{\mathrm{s}}\left[1-\left(n_{\mathrm{o}}\right)_{\mathrm{s}}\right]}=\pi_{\mathrm{s}} \varkappa_{\mathrm{s}} .
$$

Gl. (39) bzw. (40) beschreibt den Zusammenhang zwischen der Materialentnahme und der Entnahme zweiter Art für die $s$-te Stufe. 
Führt man (40) in (29) ein, so resultiert für $\pi_{\mathrm{s}} \ll 1$

$a_{\mathrm{s}}=\frac{\varepsilon+\pi_{\mathrm{s}}}{\left(\varepsilon-\chi_{\mathrm{s}} \pi_{\mathrm{s}}\right) A^{-\left(1+\frac{\alpha \pi_{\mathrm{s}}}{\varepsilon}\right)}+\left(1+\chi_{\mathrm{s}}\right) \pi_{\mathrm{s}}}$.

Da $\varkappa_{\mathrm{s}}$ durch das Produkt der Anreicherungsfaktoren $a$ der Stufen $1-s$ und den Molenbruch des Isotops im Ausgangsmaterial festliegt, kann man aus (41) z. B. durch Probieren das Entnahmeverhältnis $\pi_{\mathrm{s} \text {, opt }}$ gewinnen, das die Bedingung (37) erfüllt. Damit ist der Moldurchsatz gemäß

$$
L_{\mathrm{s}} \approx \frac{p}{\pi_{\mathrm{s}, \mathrm{opt}}}
$$

bekannt. Bei Vorgabe der spezifischen Kolonnenbelastung auf Grund der Bedingung maximalen Trennvermögens ist somit auch der Kolonnenquerschnitt $Q_{\mathrm{s}}$ bestimimt.

Da bei der Berechnung der optimalen Kaskade die Stufenlänge und über Gl. (38) damit die Stufenzahl vorgegeben wurde, soll in Abb. 12 noch gezeigt werden, wie der Gesamtinhalt $J_{\text {ges }}$, der Basisdurchsatz $L_{1}$ und die Gesamtlänge $Z_{\text {ges }}$ einer optimalen Kaskade mit vorgegebenem $a_{\mathrm{p}}$ von der Stufenzahl

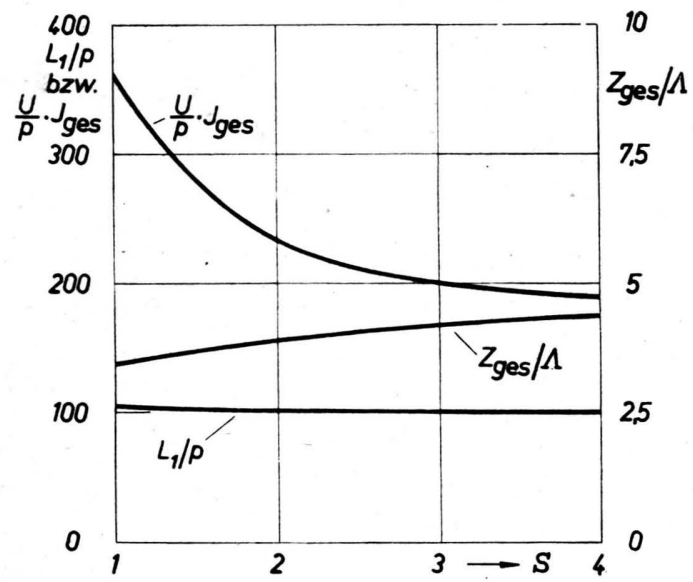

Abb. 12. Der Basisdurchsatz $L_{1}$, der Gesamtinhalt $J_{\text {ges }}$ und die Gesamtlänge $Z_{\text {ges }}$ einer optimalen Kaskade $\left(a_{\mathrm{p}}=16, n_{\mathrm{u}}=1,1 \cdot 10^{-2}\right)$ in Abhängigkeit von der Stufenzahl $S$ bei festen Werten des Entnahmestromes $p$, der Trennlänge $A$ und des Trennvermögens $U$.

Der Maßstab für $L_{1} / p$ ist mit $10 \mathrm{zu}$ multiplizieren.

${ }^{21}$ Aus (14) folgt: $J=\frac{L Z}{\Lambda U}=\frac{p}{U} \frac{L}{p} \frac{Z}{\Lambda}$; unter den angegebenen Voraussetzungen folgt daraus

$$
J_{\text {ges }}=\frac{p}{U} \sum_{s=1}^{S} \frac{L_{\mathrm{s}} Z_{\mathrm{s}}}{p \Lambda} .
$$

${ }^{22}$ Aus der allgemeinen Optimalbedingung (37) leitet man ab, daß bei nahezu kontinuierlicher Staffelung $(S \gg 1) A_{\text {ges }}=a_{\mathrm{p}}^{2}$ ist, eine Bedingung, die nach der abhängen. Dabei wird die Trennlänge $\Lambda$ und das Trennpotential $U$ für alle Stufen als konstant angenommen. Um zu einer von der Produktionsstromstärke und von den Trenneigenschaften der Kolonne unabhängigen Aussage zu gelangen, wird $L_{1}$ auf $p, Z_{\text {ges }}$ auf $\Lambda$ und $J_{\text {ges }}$ auf $p / U$ normiert ${ }^{21}$. Man erkennt, daß die Gesamtlänge mit der Stufenzahl etwas ansteigt ${ }^{22}$, während der Gesamtinhalt relativ stark abfällt; gleichzeitig ist eine sehr geringe Verminderung des Basisdurchsatzes und damit des Chemikalienverbrauches zu beobachten.

Als wesentlichste Aussage entnehmen wir der Abb. 12, daß eine Erhöhung, der Stufenzahl über $S=3-4$ hinaus den Gesamtinhalt und damit die Anlaufzeit der Kaskade kaum noch vermindert. Da jede Vergrößerung von $S$ einen zusätzlichen technischen Aufwand bedingt (s.o.), dürfte die günstigste Stufenzahl im gewählten Beispiel etwa bei $S=4$ liegen.

Abschließend wird nach dem geschilderten Verfahren eine vierstufige ${ }^{13} \mathrm{C}$-Trennanlage berechnet, die tatsächlich ausgeführt und experimentell untersucht worden ist. Die Anlage wurde für eine Produktion von $0,18 \mathrm{Mol}$ Kohlenstoff pro Tag mit etwa $12 \%{ }^{13} \mathrm{C}\left(a_{\mathrm{p}} \approx 12,4\right)$ dimensioniert.

Zunächst müssen auf Grund der in Abschnitt A 5 beschriebenen Einzelversuche die Betriebsbedingungen (Gasdruck, NaOH-Konzentration, spezifische Kolonnenbelastung) festgelegt werden:

Um die Menge der gelösten Blausäure und damit die Polymerisation zu vermindern, wurde am unteren Ende der Kaskade ein Gasdruck von 115 Torr vorgesehen, obwohl dadurch der mittlere Druck in der Kaskade mit 90-95 Torr (s. u.) merklich kleiner ist als der aus Abb. 5 abzuleitende Optimaldruck.

Da die Maxima von $U$ in Abb. 9, die eine spezifische Natronlaugebelastung von $\approx 160 \mathrm{~cm}^{3} / \mathrm{h} \cdot \mathrm{cm}^{2}$ vorschreiben, relativ flach sind, kann man zur Verkürzung der Trennlänge (vgl.Abb. 8) zu etwas geringeren spezifischen Kolonnenbelastungen übergehen $\left(\approx 135 \mathrm{~cm}^{3} / \mathrm{h} \cdot \mathrm{cm}^{2}\right)$, ohne dadurch das Trennvermögen wesentlich $\mathrm{zu}$ vermindern.

Die Natronlaugekonzentration wurde mit 4,5 $\mathrm{Mol} /$ Ltr. verhältnismäßig niedrig gewählt, um das Ausfallen von $\mathrm{Na}_{2} \mathrm{CO}_{3}$ am Produktionsende der Anlage zu vermeiden ${ }^{23}$.

Berechnung von Cohe $\mathbf{n}^{3}$ auch für eine kontinuierlich gestaffelte Anlage mit minimalem ,,Gesamtdurchsatz“" gilt. Bei kontinuierlicher Staffelung beträgt demnach die Gesamtlänge $Z_{\text {ges }}=\Lambda 2 \ln a_{\mathrm{p}}$. Im Gegensatz dazu ist bei $S=1$, d. h. bei einer. ungestaffelten Anlage $A=2 a_{\mathrm{p}}-1$ bzw. $Z=\Lambda \ln \left(2 a_{\mathrm{p}}-1\right)$.

${ }^{23} \mathrm{Das}_{\mathrm{Na}} \mathrm{CO}_{3}$ entsteht vorwiegend am oberen Ende der Kaskade aus der Kohlensäure, die im HCN-Erzeuger aus den Verunreinigungen des Ausgangsmaterials gebildet wird (vgl. die folgende Arbeit). 
Obwohl die zu diesen speziellen Betriebsbedingungen gehörende Trennlänge wegen des erwähnten Wandeffektes in geringem Maße vom Kolonnenquerschnitt und wegen des Druckgefälles längs der Kaskade von der Stellung der betrachteten Kolonne innerhalb der Anlage abhängt, wurde für die Frmittlung der Kolonnenzahl vorläufig eine mittlere Trennlänge von $3,90 \mathrm{~m}$ zugrunde gelegt.

Die Länge der einzelnen Rohrabschnitte lag aus räumlichen Gründen zu 2,6 m fest, wodurch sich pro Abschnitt ein vorläufiger Anreicherungsfaktor $A=$ 2,23 ergibt. Mit Hilfe der Optimalbedingung (37) folgt daraus für eine 2,6 m lange Stufe: $a=1,62$. Die für $a_{\mathrm{p}} \approx 12,4$ erforderliche Stufenzahl $S$ ergibt sich dann aus der Bedingung (38) zu $5-6$. Nun zeigt eine Überschlagsrechnung, daß der Durchmesser der letzten Stufe bei der vorgesehenen Entnahmestromstärke für $S=5$ nur noch etwa $8 \mathrm{~mm}$ beträgt. Da ein so kleiner Kolonnendurchmesser aus rektifikationstechnischen Gründen (Wandeffekt, Füllkörpergröße, s. o.) nicht mehr in Frage kommt, und nach Abb. 12 eine Erhöhung der Stufenzahl über $S=4$ hinaus kaum noch einen Vorteil bringt, beschränken wir uns auf vier Stufen, von denen die drei ersten aus je einem, die vierte aber aus zwei Rohrabschnitten von je 2,6 m
Länge bestehen. Für diese Kaskade berechnet man mit der mittleren Trennlänge von 3,90 m einen Anreicherungsfaktor $a_{\mathrm{p}}=12,7$.

Bei der Berechnung der Durchsätze und Querschnitte der einzelnen Stufen berücksichtigen wir nun die oben unterdrückte geringfügige Veränderung der Trennlänge längs der Kaskade. Das ist möglich, da mit der Gesamtlänge der Anlage auch der Druckabfall bekannt ist (vgl. Abb. 8) und die zu erwartenden Kolonnenquerschnitte durch eine Überschlagsrechnung abgeschätzt werden können. Man erhält so die in Tab. 3 angegebenen Werte für $A, A$ und $a$. Man berechnet nun die Endkonzentrationen $n_{0}$ der einzelnen Stufen, daraus $\varkappa$ nach Gl. (40) und gewinnt schließlich aus (41) die Entnahmeverhältnisse $\pi$, die bei vorgegebener Entnahmestromstärke $p$ die Durchsätze $l$ bzw. $L$ liefern. Die entsprechenden Natronlaugestromstärken $L_{\mathrm{NaOH}}$ sind wegen der gelösten Blausäure um $\approx 10 \%$ niedriger anzusetzen. Daraus erhält man mit der vorgesehenen $\mathrm{NaOH}-\mathrm{Konzentration}$ von 4,5 Mol/Ltr. die pro Stunde insgesamt auf die einzelnen Stufen $\mathrm{zu}$ fördernden $\mathrm{NaOH}-$ Volumina $L_{\mathrm{v}}$, woraus sich mit der spezifischen $\mathrm{NaOH}-B$ elastung von $\approx \dot{135} \mathrm{~cm}^{3} / \mathrm{h} \cdot \mathrm{cm}^{2}$ die Rohrdurchmesser $d$ ergeben. Damit sind alle Daten der Kaskade bekannt (vgl. Tab. 3).

\begin{tabular}{|c|c|c|c|c|c|c|c|c|c|}
\hline $\begin{array}{l}\text { Stufe } \\
\text { Nr. }\end{array}$ & $\begin{array}{c}A \\
{[\mathrm{~m}]}\end{array}$ & $\begin{array}{c}Z \\
{[\mathrm{~m}]}\end{array}$ & $A$ & $a$ & $\pi \cdot 10^{2}$ & $\begin{array}{c}L \\
{[\mathrm{Mol} / \mathrm{h}]}\end{array}$ & $\begin{array}{c}L_{\mathrm{NaOH}} \\
{[\mathrm{Mol} / \mathrm{h}]}\end{array}$ & $\begin{array}{c}L \\
{\left[\mathrm{~cm}^{3} / \mathrm{h}\right]}\end{array}$ & $\begin{array}{c}d \\
{[\mathrm{~mm}]}\end{array}$ \\
\hline 1 & 3,60 & 2,60 & 2,50 & 1,75 & 0,130 & 5,77 & 5,2 & 1160 & 32,8 \\
\hline 2 & 3,75 & 2,60 & 2,36 & 1,68 & 0,242 & 3,10 & 2,8 & 625 & 24,2 \\
\hline 3 & 3,90 & 2,60 & 2,24 & 1,62 & 0,461 & 1,63 & 1,47 & 325 & 17,4 \\
\hline 4 & $.4,10$ & 5,20 & 4,25 & 2,63 & $1,04_{5}$ & 0,72 & 0,65 & 145 & 11,6 \\
\hline
\end{tabular}

Tab. 3. Die Daten einer ,optimalen“ vierstufigen Kaskade, die pro Tag 0,18 Mol Kohlenstoff mit $12 \%{ }^{13} \mathrm{C}$ liefern soll.

\title{
Erfahrungen mit einer chemischen Austauschanlage zur Anreicherung von ${ }^{{ }^{3} \mathrm{C}} \mathrm{C}$
}

\author{
Von E. W. Becker, K. Bier, S. Scholz und W. Vogell
}

Aus dem Physikalischen Institut der Universität Marburg a. d. Lahn

(Z. Naturforschg. 7 a, 664-668 [1952]; eingegangen am 4. Juni 1952)

Es wird die praktische Ausführung der in der voranstehenden Arbeit berechneten vierstufigen Trennkaskade beschrieben. Die Anlage liefert bei der vorgesehenen Produktionskonzentration von $12 \%{ }^{13} \mathrm{C}$ pro Tag 0,16 Mol Kohlenstoff, während der Berechnung eine Produktionsleistung von 0,18 Mol zugrunde gelegt wurde. Die Produktionskonzentration von $12 \%{ }^{13} \mathrm{C}$ wird nach einer Anlaufzeit von nur 53 Stdn. erreicht.

$\mathrm{I}^{\mathrm{n}}$ n der vorausgehenden Arbeit ${ }^{1}$ wurde gezeigt, wie sich auf Grund experimentell gewonnener Daten die Abmessungen einer chemischen Austauschanlage $^{2}$ angeben lassen, die hinsichtlich bestimmter Gesichtspunkte ,optimal“" arbeitet. Im Zahlenbeispiel wurden die Dimensionen einer Apparatur fest-

${ }^{1}$ E.W. Becker u. K. Bier, Z. Naturforschg. 7a, 651 [1952]. gelegt, die nach möglichst kurzer Anlaufzeit 0,18 Mol Kohlenstoff pro Tag mit $12 \%{ }^{13} \mathrm{C}$ liefern soll. Im folgenden werden die praktische Ausführung dieser Anlage und die mit ihr gewonnenen Erfahrungen beschrieben.

- C. A. Hutchinson, D. W. Stewart u. H. C. Urey, J. chem. Physics 8, 532 [1940]. 